\title{
Marché international du soja : des flux en profonde recomposition
}

The international soybean market: far-reaching changes in flows

Oléagineux, Corps Gras, Lipides. Volume 8, Numéro 3, 191-8, Mai - Juin 2001, La filière

Auteur(s) : Marie-Hélène DABAT, Xiande LI, Frédéric LANCON, Cirad CA-Calim, TA 70/16, 73, rue J.-F. Breton, 34398 Montpellier Cedex 5, France.

Résumé : Le soja est le principal oléo-protéagineux produit et échangé au monde. Les flux sont polarisés autour de trois grands ensembles géographiques : l'Amérique (États-Unis, Argentine, Brésil), I'Europe occidentale et les pays asiatiques (Chine, Inde, Japon et NPI). La production et la transformation du soja ont fortement crû dans les années 90 sous l'effet de la poussée des pays américains. Le commerce des graines a tendance depuis peu à augmenter plus que celui des principaux produits transformés (tourteau, huile), tiré par les achats asiatiques. Malgré les qualités reconnues au soja (diversité des produits, qualités nutritionnelles, protection écologique), les perspectives d'avenir de son marché et la recomposition des échanges internationaux sont largement dépendantes de l'émergence de nouveaux pays fortement producteurs ou consommateurs, de l'évolution de l'utilisation des OGM et des farines animales et des conséquences de l'entrée de la Chine dans l'OMC.

Mots-clés : oléagineux, soja, tourteau, huile, commerce international.

Summary : Soya is the oil and protein crop most grown and traded in the world. Movements are centred on three main geographical ensembles: America (the USA, Argentina and Brazil), Western Europe and the Asian countries (China, India, Japan and NICs). Soybean production and processing increased strongly in the 1990s under the impetus of the American countries. The grain trade has recently tended to increase more strongly than that of the main processed products (cake and oil), as a result of Asian buying. In spite of the recognised qualities of soybean (product diversity, nutritional qualities, ecological conservation), the future prospects of the soya market and the recomposition of international trade are strongly dependent on the emergence of new strong production or consumer countries, the evolution of the use of GMOs and animal meal and the consequences of China joining the WTO.

Keywords : oil crops, soybean, meal, oil, international trade. 


\section{ARTICLE}

Le marché international du soja, principal oléoprotéagineux cultivé et échangé dans le monde même si l'essentiel des récoltes reste dans les pays producteurs, est à la croisée des chemins. Loué à la fois pour ses qualités nutritionnelles (fort contenu en protéines et acides aminés) et son impact écologique (faible besoin en engrais et produits phytosanitaires), le soja est largement diabolisé en tant que principal produit agricole génétiquement modifié. L'évolution à venir du marché international du soja apparaît aujourd'hui d'autant plus incertaine qu'il est actuellement concentré entre un nombre réduit de pays, parmi lesquels des puissances économiques telles que les ÉtatsUnis, la Chine et l'Union européenne.

\section{Caractéristiques structurelles du marché du soja}

\section{Premier oléagineux dans les échanges mondiaux malgré une très forte utilisation locale}

Le soja représente plus de la moitié de la production mondiale de graines oléagineuses. Il domine aussi le commerce international de ces graines en constituant plus des deux tiers des volumes exportés (figure 1). Il pourvoit aux deux tiers de la production mondiale de tourteaux et à plus de 68 $\%$ des exportations (figure 2). Le soja fournit enfin $28 \%$ du volume de la production mondiale d'huile et représente $21 \%$ du volume des exportations (figure 3). Si sa position centrale sur le marché des tourteaux est incontestable, le soja doit affronter, sur le marché de l'huile, la concurrence de l'huile de palme et de colza (respectivement 22 et $14 \%$ de la production mondiale).

Malgré son importance au niveau international, seulement $29 \%$ de la production de graines de soja est vendue sur le marché mondial, soit 46 millions de tonnes (Mt) pour la campagne 1999/2000. Si I'on considère la part respective de chacun des produits dérivés (huile et tourteau) et des graines dans les échanges du " complexe soja ", on peut estimer qu'en équivalent graines (sur la base de $0,18 \mathrm{~kg}$ d'huile et $0,80 \mathrm{~kg}$ de tourteau pour $1 \mathrm{~kg}$ de graine), le tourteau représente actuellement $36 \%$ des échanges, alors que les commerces des graines et de l'huile représentent respectivement 34 et $30 \%$. Le commerce du tourteau a tendance à diminuer tandis que celui des graines augmente proportionnellement aux autres produits issus du soja.

\section{Grande diversité des produits alimentaires à base de soja}

Les produits joints, huile et tourteau, représentent le débouché majeur du soja (environ $90 \%$ de la production mondiale est destinée à la trituration). Les produits dérivés de l'huile et du tourteau entrent dans la fabrication d'une large gamme de produits alimentaires et chimiques (alimentation animale, huile raffinée, acide gras, stérol, lécithine, farine, etc.).

L'utilisation de la graine entière (après broyage et filtration) pour l'alimentation humaine reste essentiellement concentrée dans les pays asiatiques (Chine, Japon, Corée, Indonésie) où elle entre dans la fabrication de sauces, de fromages à base de lait de soja et de produits fermentés. 
Depuis une quinzaine d'années, la fabrication de produits à base de soja se développe dans l'industrie agro-alimentaire des pays industrialisés (Canada, Suède, France) du fait notamment de la bonne image que véhicule le soja auprès du consommateur occidental (santé). Bien qu'en très forte croissance, ce marché ne représente qu'une part infime des utilisations totales.

Des marges plutôt réduites dans la trituration du soja ont conduit récemment l'industrie des huiles à rechercher des possibilités de meilleure rentabilisation de leurs équipements. Une des voies explorées est celle de la fabrication de concentrés de protéines de soja pour la consommation humaine et autres usages industriels, notamment la nourriture animale. Plus de $75 \%$ des concentrés de protéines de soja sont valorisés pour l'alimentation humaine, le reste étant utilisé pour les animaux domestiques et en remplacement du lait pour les jeunes veaux et cochons.

La demande de concentrés de soja se développe en Europe de l'Est et dans les pays en développement à la place ou en substitution des produits à base de viande. Ce marché devrait atteindre 500000 tonnes par an d'ici une décennie. Il augmente de plus de $15 \%$ par an. Son extension dépendra de plusieurs facteurs : la disponibilité des savoir-faire et des technologies de transformation, le développement de l'industrie agro-alimentaire, les politiques nationales des pays producteurs, l'acceptation par les consommateurs de tels produits et la disponibilité locale de protéines alternatives.

Il faut noter enfin le développement de procédés fondés sur une cuisson rapide des graines pour en retirer tous les éléments anti-nutritionnels et, ainsi, les utiliser directement pour l'alimentation animale (Full Fat Soybean).

\section{Polarisation des échanges entre une poignée de pays}

Les échanges mondiaux de grain, de tourteau et d'huile de soja sont polarisés autour de trois grands ensembles géographiques (figures 4 et 5 ) :

- un groupe comprenant les États-Unis, l'Argentine et le Brésil ;

- un autre constitué par l'Europe occidentale ;

- un troisième comprenant la Chine, I'Inde, le Japon, et les pays asiatiques nouvellement industrialisés ou en voie d'industrialisation rapide.

Les États-Unis produisent un peu moins de la moitié (72 Mt en 2000) de la production mondiale (159 $\mathrm{Mt})$. Le Brésil (33 Mt) et l'Argentine (21 Mt) constituent le deuxième groupe de producteurs. La Chine reste un producteur important avec une production annuelle qui varie entre 13 et $16 \mathrm{Mt}$ (voir dossier) dont une infime partie est exportée sur le marché asiatique (figure 6).

Les États-Unis dominent les échanges de graines même si leur part est en diminution ( 57 \% des exportations mondiales) devant le Brésil (24\%), alors que l'offre d'huile et de tourteaux est beaucoup plus diversifiée géographiquement compte tenu de la répartition des capacités de trituration. L'Argentine, le Brésil, I'Union européenne et les États-Unis sont les principaux fournisseurs d'huile à l'échelle mondiale. Les mêmes pays sont également les principaux vendeurs de tourteaux auxquels vient s'ajouter l'Inde qui joue un rôle très actif sur le marché asiatique en forte croissance. 
L'Union européenne (Pays-Bas, Allemagne, Espagne, etc.) a perdu sa place de principal acheteur de graines de soja sur le marché mondial en 1998 (environ 16 Mt par an actuellement), cédant cette place à l'Asie qui représente désormais le premier marché avec un volume qui dépasse les $22 \mathrm{Mt}$ en 2000, en raison de la forte progression de la demande dans les pays nouvellement industrialisés et dans les marchés émergents (Japon, Taïwan, Corée du Sud, Indonésie) et plus récemment en Chine. L'utilisation des tourteaux est concentrée elle sur trois pôles que sont les États-Unis, I'Union européenne et les pays asiatiques (environ $27 \mathrm{Mt}$ pour chacun de ces pôles). La consommation asiatique a beaucoup augmenté depuis le début des années 90 . Le marché de I'huile a été marqué par la croissance des importations chinoises qui ont plus que triplé depuis le début de la décennie puis sont retombées en dessous de $1 \mathrm{Mt}$ à partir de 1998-1999 (voir dossier).

\section{Tendances récentes des échanges mondiaux}

\section{Croissance accélérée de la production mondiale dans les années 90}

Mise à part une mauvaise récolte en 1995, la production de soja est en progression continue sur la décennie 90 (tableau 1). Les prévisions les plus récentes estiment que la production de la campagne 2000/2001 devrait atteindre un nouveau record avec une récolte avoisinant les $169 \mathrm{Mt}$.

Cette forte croissance de la production est due à une augmentation des superficies ensemencées en soja aux États-Unis et en Argentine et à des rendements élevés au Brésil et en Argentine relatifs à des conditions agro-climatologiques très favorables. Les productions chinoise et indienne, élevées, ne font que se maintenir.

\section{Marché des grains : reprise des exportations des États-Unis et boom des importations chinoises}

Le marché des grains a presque doublé au cours de la dernière décennie, passant de $25 \mathrm{Mt}$ à plus de $47 \mathrm{Mt}$, soit une progression supérieure à celle de la production (+ $55 \%$ sur la même période) (tableaux 2 et 3 ).

Après un ralentissement de la compétitivité du soja nord-américain dans les années 1998-1999, dû à la bonne tenue des exportations latino-américaines liée notamment à la dévaluation de la monnaie brésilienne, les États-Unis devraient atteindre cette année un volume d'exportation jamais atteint par ce leader mondial. Les deux tiers du soja cultivé aux États-Unis sont exportés. Les ventes des États-Unis se dirigent à $31 \%$ vers le Mexique (malgré les concurrence brésilienne, argentine et canadienne), à $8 \%$ vers les pays asiatiques et à $6 \%$ vers I'Union européenne.

Le Mexique est devenu au cours des deux dernières années un important importateur de soja américain car son économie se développe et le pays bénéficie d'une réduction substantielle de tarif liée à l'accord NAFTA avec les États-Unis. Le Mexique tirant environ un tiers de ses revenus du pétrole et les prix du brut ayant presque triplé récemment, le pays a investi dans ses autres industries et notamment dans l'industrie porcine et celle de la trituration, ce qui a stimulé les achats de matières premières agricoles aux États-Unis voisins. 
En revanche, les exportations des États-Unis vers l'Union européenne ne progressent pas du fait de plusieurs facteurs valables pour l'ensemble des fournisseurs des pays européens : substitution du colza au soja dans ces pays (en partie produit localement : bonnes récoltes en 1998 et 1999), substitution de céréales moins onéreuses au soja dans la composition des nourritures animales (surtout à partir de 2000), affaiblissement de l'euro.

La reprise des économies asiatiques, liée notamment à la dévaluation de leurs monnaies, a accéléré l'élevage de volailles (marché intérieur et exportation) et les importations de soja pour les nourrir. Mais au niveau des importations de grains, la tendance marquante de ces dernières années est la forte progression des achats chinois (plus de $10 \mathrm{Mt}$ en 1999/2000), qui s'est faite au détriment des achats de tourteau (division par 7 de 1997 à 2000) et d'huile (division par 4). Cette évolution est due à un revirement politique du Gouvernement chinois qui cherche à protéger l'industrie de trituration nationale des importations : mise en place d'une TVA de $13 \%$ sur le tourteau de soja importé mi1999 et contingentement des importations d'huile de soja. Sur le marché chinois, le soja subit aussi la concurrence du colza puisque la Chine aurait importé 3,7 Mt de ce produit en 1999-2000 (essentiellement d'Australie et du Canada). Les importations de grains en Chine devraient se maintenir en 2000/2001. L'augmentation des importations chinoises de grains bénéficie essentiellement aux États-Unis au détriment du Brésil et de l'Argentine plus traditionnellement exportateurs de tourteau et d'huile de soja.

Les exportations américaines sont facilitées par deux programmes qui offrent des facilités de paiement aux pays acheteurs : I'Export Credit Guarantee Program (GSM-102), qui couvre des crédits jusqu'à 3 ans, et l'Intermediate Export Credit Guarantee Program (GSM-103), qui propose ses services de crédit jusqu'à 10 ans. Elles sont encouragées également par le biais de subventions à la production, qui n'existent pas dans d'autres pays et qui tirent les prix internationaux à la baisse.

\section{Marché des tourteaux : tassement des échanges lié à l'augmentation des capacités locales de trituration}

L'amélioration de la production agricole et l'accroissement rapide des capacités techniques (mise en production de nouvelles unités industrielles, modernisation d'installations plus anciennes) ont permis au Brésil et surtout à l'Argentine d'augmenter le volume de soja réservé à la trituration et à l'exportation dans la deuxième moitié des années 90 (tableau 4).

L'Argentine, déjà premier exportateur d'huile de soja, est devenue également le premier fournisseur mondial de tourteaux en dépassant le Brésil en 1997 (tableau 5), du fait de la rapide expansion des superficies cultivées et du doublement de ses capacités de trituration en une décennie, mais aussi d'une demande intérieure limitée et d'une politique fiscale qui favorise les exportations de produits transformés. Dernier facteur et non des moindres, l'Argentine bénéficie d'un avantage comparatif par rapport au Brésil qui est la distance réduite entre les zones de culture du soja, la localisation des usines de trituration et les ports d'exportation, ce qui limite les coûts de transport à la fois du soja et des produits transformés. 
L'accroissement de l'activité de trituration en Chine est beaucoup plus récente. On note dans ce pays un secteur de la trituration à deux vitesses : d'anciennes entreprises publiques obsolètes et déficitaires, d'une part, et un sous-secteur récent et performant localisé près des ports, d'autre part. Ces dernières entreprises utilisent du soja importé qu'elles triturent essentiellement pour le marché intérieur (voir autre article).

Le bon niveau des exportations de tourteaux d'Argentine et du Brésil permet à ce marché de se maintenir malgré la stagnation des exportations en provenance des États-Unis et d'Europe et la réorientation de la production chinoise vers le marché intérieur.

L'Inde connaît des variations de son niveau d'exportation liées à l'insuffisante qualité du produit offert. Les fournisseurs indiens ont cependant fait des efforts commerciaux d'amélioration de la qualité du soja national à partir de 1998, après la mise à l'écart par certains acheteurs exigeants, notamment sud-coréens.

Du côté de la demande, la baisse drastique des importations chinoises, à laquelle les experts ne s'attendaient pas, a été compensée par le bon niveau des importations européennes, tirées par I'Espagne, et la hausse modérée mais continue des achats de pays d'Afrique du Nord et du MoyenOrient (tableau 6).

On note un ralentissement de la consommation de tourteau dans les années 1998-1999, dû à plusieurs facteurs qui s'atténuent cependant (tableau 7). En Europe, le marché de la viande s'est ralenti du fait de différents incidents sanitaires. De plus, la baisse du prix des céréales rend moins attrayant l'élargissement de l'utilisation du soja dans la formulation des aliments comme source de calorie. II revient à sa fonction première de pourvoyeur de protéines. En Asie, la récession économique des années 1996-1998 a entraîné un ralentissement de la croissance, une baisse de la consommation de protéines animales et, partant, des besoins en aliment pour l'élevage.

Mais ce ralentissement général de la demande de tourteau a été atténué par la baisse tendancielle de son prix qui en fait une source de protéines intéressante sur le marché face à des alternatives de plus en plus coûteuses (farine de poisson) ou déconsidérées (farines animales). On peut s'attendre à un accroissement des importations de soja dans I'Union européenne lié à la découverte de I'ESB (encéphalopathie spongiforme bovine), par substitution partielle de soja aux 2,5 Mt de farines animales utilisées jusqu'ici (ce qui représente un équivalent de 2,9 Mt de tourteau de soja). Mais les importations de soja (grain et tourteau) ne seront pas seules à se substituer aux farines animales décriées. On s'attend à une progression de l'utilisation de tourteaux locaux et importés de colza et de tournesol, de céréales locales, de gluten de maïs importé, enfin de pois et haricots locaux et importés. Cette compétition entre sources d'approvisionnement pour la nourriture animale devrait de plus avoir lieu dans un contexte d'inflexion des prix du tourteau de soja et de fragilité de la demande de bœuf par les consommateurs européens.

Côté asiatique, la stagnation des ventes de tourteaux devrait être enrayée en 2001 par l'augmentation de la consommation, en particulier en Chine, liée à la croissance de la production de protéines animales. 


\section{Marché des huiles : concurrence des autres oléagineux mais bonne tenue du marché}

L'évolution de l'offre d'huile est intrinsèquement liée à celle des tourteaux (tableau 8). Au niveau de la demande, on note une stabilité des achats des principaux importateurs (Iran, Maroc, Pakistan), une réduction des importations chinoises (voir dossier) et une progression des achats de I'Inde (multiplication par 3 des importations entre 1997 et 2000) qui a augmenté aussi sa propre production face à des besoins qui progressent vite (tableau 9), faisant de ce pays le quatrième utilisateur mondial d'huile de soja derrière les États-Unis, le Brésil et la Chine (tableau 10).

Le Pakistan voit lui aussi ses besoins en huile augmenter très vite. II prévoit, pour stimuler l'accroissement de sa capacité de trituration, de favoriser la production agricole de grains à fort contenu en huile comme le tournesol et de faire varier ses droits de douane de façon à décourager l'importation directe d'huile (de palme et de soja) et encourager celle de grains.

Sur une plus longue période, la consommation d'huiles comestibles, en particulier de soja, est en augmentation régulière sous l'effet de la croissance démographique de plusieurs pays, de l'amélioration des niveaux de vie et de la modification des modes de consommation, ce que ne reflète pas forcément la stabilité des importations. La consommation d'huiles et de graisses est passée en Chine de 5-6 kg par an dans les années 80 à environ $10 \mathrm{~kg}$ actuellement dont $8 \mathrm{~kg}$ de produits végétaux.

\section{Baisse des prix du soja et de l'huile, appréciation des tourteaux}

Une des particularités du soja est le caractère lié des produits dérivés que sont le tourteau et l'huile. La production de chacun de ces produits est inhérente à la fabrication de l'autre. De ce fait, le calcul de la marge totale du triturateur va dépendre de l'état des prix de ces deux produits joints sur leurs marchés respectifs.

La baisse de la production d'huile de palme pendant deux années, notamment en Indonésie sous l'effet d'aléas climatiques et d'une politique commerciale restrictive, a entraîné une hausse générale des cours des huiles végétales, substituables entre elles, en 1998 (figure 7). Le prix des huiles a ensuite brutalement chuté de 100 US\$ environ par tonne en 1999 (soja, coton, tournesol, colza) et a continué sa baisse par la suite du fait de l'accroissement des productions d'huile de palme (Indonésie) et de colza (Union européenne).

Le prix du tourteau de soja s'était lui stabilisé à 150 \$ la tonne en 1999 et 2000 après avoir avoisiné les 250 \$ en 1996/1997. Son prix devrait remonter à presque 200 \$ en 2001 sous l'effet de la reprise du marché de l'aliment pour bétail en Asie mais aussi en Europe où la demande de protéines et de graisses végétales est stimulée du fait des problèmes récents de qualité des farines animales.

Paradoxalement, l'accroissement de la demande de tourteau de soja en Europe a actuellement un effet très négatif sur le marché des huiles végétales, toutes origines confondues (soja, palme, coco, etc.). L'excédent déjà important de ce marché ne peut que s'accroître et les cours de ces huiles dégringoler encore. Les conséquences sont moins graves pour les triturateurs de soja - qui peuvent se rattraper sur le tourteau - que pour les producteurs d'huile de palme (Malaisie, Thaïlande, Indonésie) - qui ne peuvent eux compenser cette perte. 
Concernant le grain de soja, son faible niveau de prix se maintient. Ceci peut être attribué à la dévaluation de la monnaie brésilienne début 1999, qui a rendu les exportations de ce pays très compétitives au sein d'un marché ouvert, et à un meilleur approvisionnement du marché du fait de l'accroissement des superficies plantées en soja. Ce prix plancher peut s'expliquer aussi par une mise sur le marché de plus grandes quantités de tourteau indien à un prix très intéressant, qui oblige d'ailleurs les exportateurs à réduire leurs marges de façon importante.

De manière générale, les activités de trituration du soja se portent plutôt bien et montrent un retour à l'équilibre en 2000/2001 en comparaison à la saison précédente. Les experts constatent cependant une érosion des marges de cette activité liée à la baisse du prix de l'huile de soja.

\section{Grands débats et perspectives d'avenir}

\section{Apparition du soja génétiquement modifié dans les flux mondiaux}

Le débat sur les OGM ajoute un élément de concurrence entre pays selon qu'ils sont utilisateurs ou non de semences génétiquement modifiées. Le soja représente actuellement la part la plus importante de la production mondiale d'OGM, soit $54 \%$ contre $28 \%$ pour le maïs et $9 \%$ pour le coton ou le colza. Moins de $10 \%$ de la production brésilienne de soja serait actuellement GM, par opposition avec l'Argentine où environ 80-90 \% des semences utilisées le seraient sans pour autant rencontrer des difficultés de commercialisation. Aux États-Unis, on évalue le soja GM à $65 \%$ des récoltes.

Au Brésil, la résistance aux OGM s'explique par la prise de conscience du refus des consommateurs européens (Pays-Bas, Italie, France, Espagne, Royaume-Uni, etc.) et japonais d'acheter des produits $\mathrm{GM}$. Alors que les acheteurs de soja réfractaires organisent leur protection (nouvelle loi du Parlement européen sur les produits GM en février 2001, nouvelles lois sur la labellisation en Corée du Sud et au Japon entrées en application respectivement le $1^{\mathrm{er}}$ avril et le $1^{\mathrm{er}}$ mars 2001), la position des autorités brésiliennes n'est pas consensuelle à ce sujet. Un des États en pointe dans ce combat contre les OGM est le Rio Grande do Sul (I'un des trois grands États producteurs) ; il y voit là un argument commercial de taille (à la fois en faveur du soja mais aussi de la volaille exportés) malgré la faible adhésion des agriculteurs. En effet, des chaînes de grandes surfaces européennes (Carrefour, Asda, etc.) et des importateurs du Sud-Est asiatique (Corée du Sud) orientent leur approvisionnement vers le Brésil qui présente la garantie la plus sérieuse d'obtenir du soja non GM par rapport aux autres pays. Pourtant, le ministère de l'Agriculture brésilien avait donné au groupe Monsanto l'autorisation de distribuer des semences résistantes à l'herbicide Roundup Ready pour une superficie de 400000 ha. Mais l'action menée par Greenpeace, le ministère de l'Environnement et plusieurs associations de consommateurs a provoqué l'interruption de l'opération en attendant que la preuve soit apportée que les semences GM n'ont aucun effet sur l'environnement. Un moratorium de 5 années pour la non-utilisation des OGM est également à l'étude par le Parlement brésilien. 
Malgré leur caractère illégal, 800000 à 1 million d'ha auraient été plantés avec du soja GM dans les États du Rio Grande do Sul, Parana et Mato Grosso do Sul en 2000, ce qui représenterait une production de plus de $3 \mathrm{Mt}$. Les semences utilisées auraient été importées illégalement d'Argentine et du Paraguay où les cultures GM sont autorisées depuis plusieurs années. Les agriculteurs brésiliens résistent difficilement aux avantages des semences GM (moins d'herbicide nécessaire, coûts de production réduits de 15-20 \%), surtout en période de contraction des prix de vente du soja. Soixante-dix pour cent des agriculteurs brésiliens seraient favorables aux semences GM. Dans son effort de persuasion de ne pas planter de soja GM, l'État du Rio Grande do Sul a proposé à ses paysans fin 1999 d'échanger gratuitement leurs semences GM contre des semences traditionnelles. Cette opération n'a pas eu l'effet escompté car elle est intervenue trop tardivement, le semis ayant déjà démarré. Les contraintes à la stratégie non OGM du Brésil sont de plusieurs natures : il n'y a pas de prime à la qualité (même prix pour le soja GM et le soja non GM) alors que les coûts de production diffèrent, il n'est pas possible actuellement de garantir le caractère non GM du soja commercialisé ou de distinguer comme aux États-Unis les deux types de soja (nombre d'inspecteurs insuffisant, procédures inexistantes, complexité de la filière de production : le produit change de main une douzaine de fois en moyenne, coût du contrôle).

De l'autre côte de l'océan, la Chine n'est pas défavorable elle à la production de soja GM bien qu'elle en récolte très peu. Du fait de la taille de sa population, d'une ressource foncière limitée et d'une demande croissante en produits agricoles, la Chine recherche tous les moyens d'augmenter les rendements de ses cultures. Les travaux de recherche sur les variétés GM sont avancés. Le soja GM n'a pas atteint cependant l'étape de la production commerciale. Actuellement, la Chine importe librement des produits agricoles GM, en particulier des États-Unis et du Canada. Une réglementation devrait prochainement être mise en place de façon à maîtriser et à contrôler les flux de produits GM.

Enfin, le débat sur les OGM a aussi un effet sur la concurrence entre produits oléagineux. Un nombre croissant d'entreprises agro-alimentaires européennes, notamment anglaises, ont cessé d'utiliser le soja au bénéfice du colza, pour lequel l'isolement des grains GM paraît mieux garanti, afin de satisfaire les détaillants qui réclament des produits alimentaires sans OGM.

Alors que la mise au point des premières variétés de soja GM avait pour but de réduire les coûts de production et de simplifier les techniques culturales, une nouvelle vague de travaux portent sur la mise au point de semences $G M$ en vue d'utilisations industrielles spécifiques. Cette nouvelle orientation tournée vers l'utilisation finale est sans doute une des réponses possibles à la question de la traçabilité, au cœur du débat sur le soja GM en Europe. Ce nouveau champ d'innovation pourrait contribuer à répondre aux contraintes qui obèrent la hausse des rendements en milieu tropical humide.

\section{Conséquences sur le commerce mondial de l'entrée de la Chine dans l'OMC}

Les perspectives du commerce mondial du soja dépendent en partie des conséquences de l'entrée de la Chine dans I'OMC. L'intégration de cette grande puissance économique devrait favoriser l'ajustement des marchés et la restructuration de ses modes de production aussi bien agricoles qu'industriels au niveau mondial. Pour l'instant, le pays n'a fait que signer en novembre 1999 un accord bilatéral avec les États-Unis, examen de passage pour susciter l'avis favorable à son entrée dans l'OMC des autres membres de l'organisation. 
Concernant les grains et les tourteaux de soja, l'arrivée de la Chine à l'OMC ne devrait pas avoir un impact important sur la production locale et les échanges mondiaux de ces produits. Les droits de douane pour les grains et les tourteaux sont passés respectivement à 3 et $5 \%$ sans restriction quantitative d'importation bien avant la signature de l'accord avec les États-Unis et ne devraient pas être modifiés avec l'intégration de la Chine. Le commerce des grains est libéralisé depuis plusieurs années en Chine, aussi bien à l'intérieur qu'à l'extérieur du pays. II n'existe pas de quota à l'importation ni d'intervention publique au niveau de la production et des échanges. Le seul effet lié à l'OMC dans ce domaine est indirect, il pourrait venir de la libéralisation progressive du quota sur le maïs. En effet, des achats de plus en plus massifs de maïs à l'étranger pourraient avoir pour conséquence économique d'abaisser le prix intérieur du maïs et d'inciter les agriculteurs à augmenter les superficies en soja. Concernant le tourteau de soja, l'évolution des importations dépendra plus du maintien ou de la suppression de la TVA de $13 \%$ sur le tourteau acheté à l'étranger que des effets tarifaires proprement dits de l'intégration de la Chine à l'OMC.

Il en va autrement de l'impact de l'OMC sur les échanges d'huile de soja. Dans l'accord avec les ÉtatsUnis, dont l'essentiel devrait être incorporé dans l'accord OMC, la Chine s'est engagée à ouvrir progressivement son marché en mettant en place un système de quotas (TRQ's) pour le blé, le riz, le maïs, le coton et l'huile de soja, avec élargissement graduel des quotas sur une période de 5 ans. Elle accepte notamment d'importer de l'huile de soja taxée seulement à $9 \%$ - au lieu des $13 \%$ actuels jusqu'à un quota de 1,7 Mt (amené progressivement à 3,3 Mt en 2005). Les marchandises au-delà de ce quota seront importées librement mais taxées plus fortement (74 \% en 2000 en diminution jusqu'à $9 \%$ en 2006). Les importateurs privés d'huile devraient prendre une part de plus en plus importante à ce quota (50 \% en 2000 en progression jusqu'à $90 \%$ en 2005). Le système devrait être aboli en 2006 et transformé en un tarif unique de $9 \%$ pour des quantités illimitées d'huile de soja. La Chine se prépare à cette situation en accroissant et modernisant depuis plusieurs années son potentiel de trituration pour le rendre compétitif (voir dossier). Si cette restructuration n'était pas efficace, les pays exportateurs (États-Unis, Argentine, Brésil, etc.) et les importateurs chinois s'en trouveraient favorisés quand le pays aura officiellement rejoint l'OMC et au fur et à mesure que les droits de douane diminueront dans les 3-4 années à venir.

\section{CONCLUSION}

L'économie mondiale du soja évolue rapidement, entraînant une profonde recomposition des échanges internationaux de ce produit : croissance des capacités d'offre de tourteau et d'huile en Amérique latine, émergence de nouveaux pays producteurs ou triturateurs (Inde, Nigeria, etc.), augmentation des besoins de protéines pour l'alimentation animale en Asie, recherche de produits de substitution aux farines carnées dans l'alimentation animale en Europe, renforcement de l'exigence des consommateurs des pays développés. Mais plus que tout, les contours à venir du marché mondial du soja (grain, tourteau, huile) dans un contexte de libéralisation des échanges internationaux dépendront de l'évolution des modes alimentaires et de la compétitivité de la filière en Chine. Ce pays est en effet à la fois un producteur non négligeable et le principal consommateur de soja au monde. 


\section{REFERENCES}

AgExporter, décembre 2000.

Agricultural Outlook, March 2000 and September 2000.

DABAT MH, LANÇON F. Fiche produit du CIRAD 1999 : marché international du soja.

Oils \& Fats International, May 2000.

Public Ledger, plusieurs numéros.

USDA, Oilseeds : World Markets and Trade, January 2000 to December 2000.

\section{Illustrations}

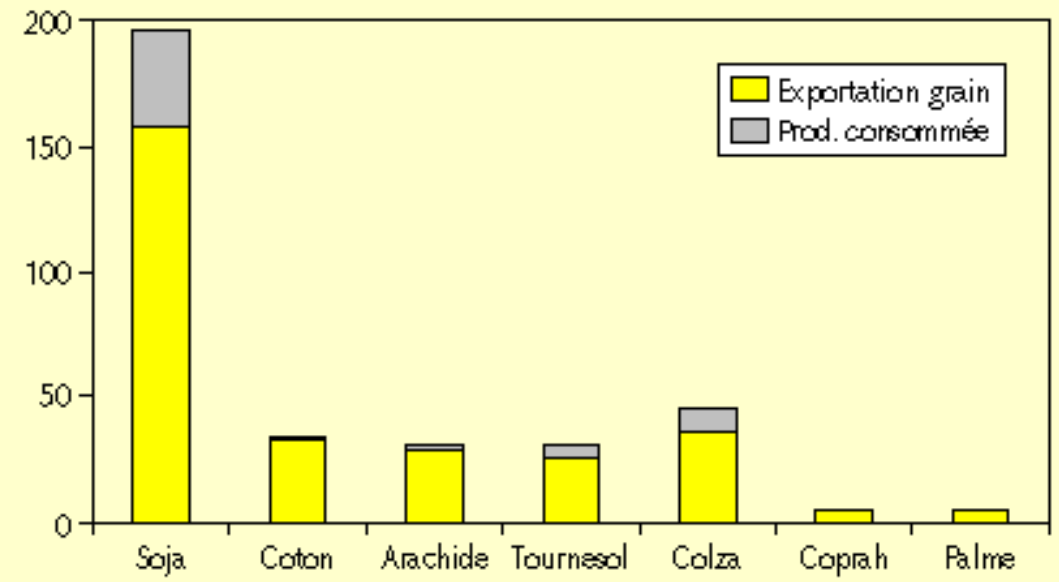

Figure 1. 


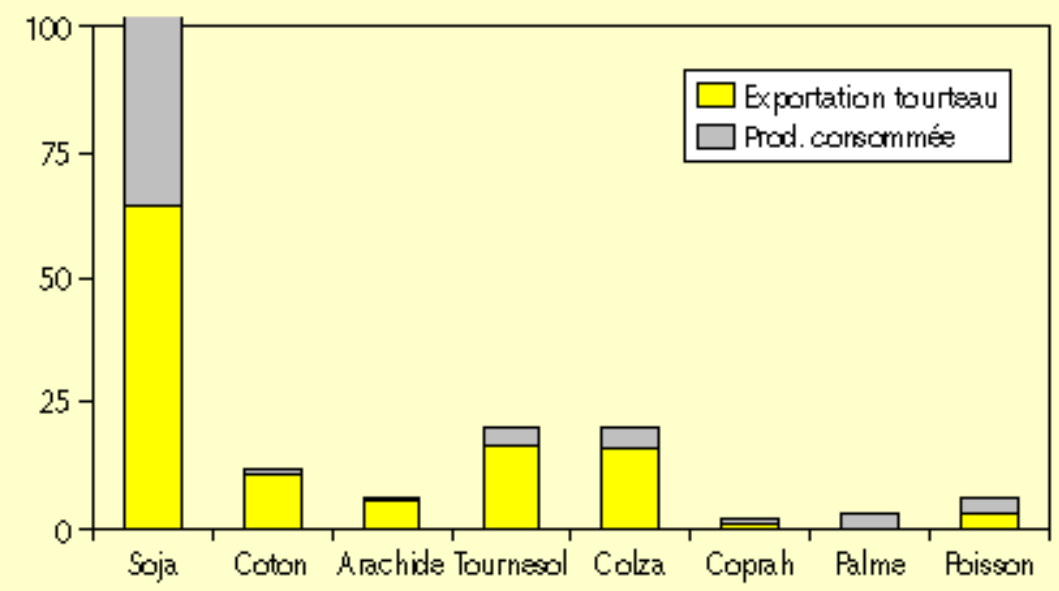

Figure 2 .

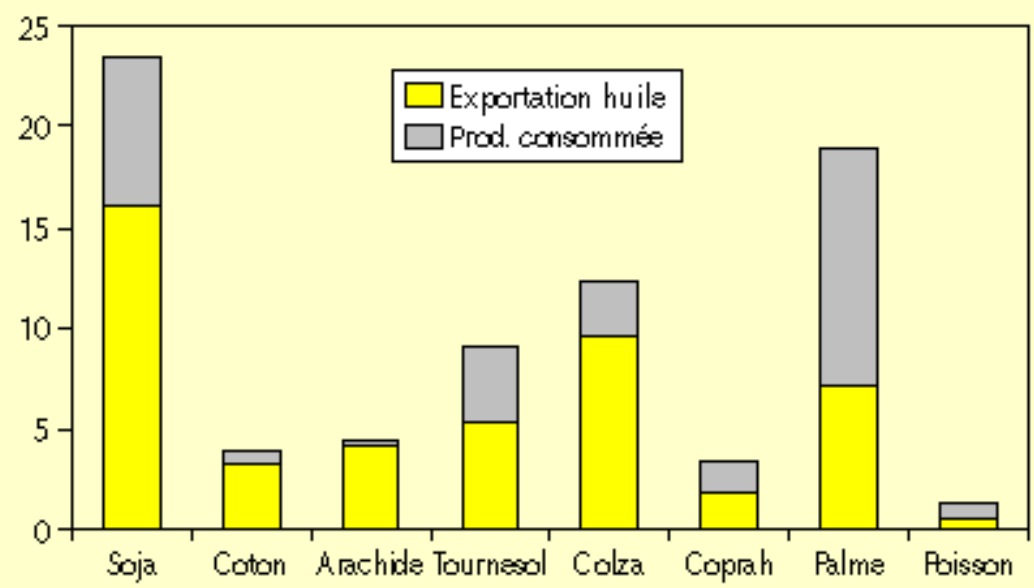

Figures 1, 2 et 3. Place du soja parmi les oléagineux en 1998/1999, en millions de tonnes $=M t$ ( $d^{\prime}$ 'après FAS-USDA).

Remarque : les échelles des graphiques sont différentes. 


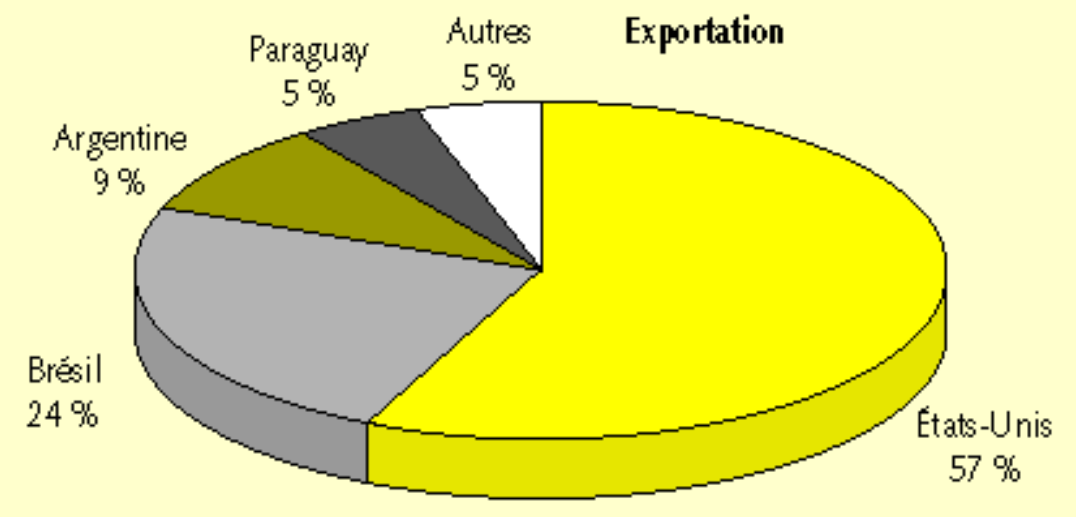

Figure 4. Répartition des flux de soja entre principaux pays en 2000 à l'exportation (d'après FAS-USDA).

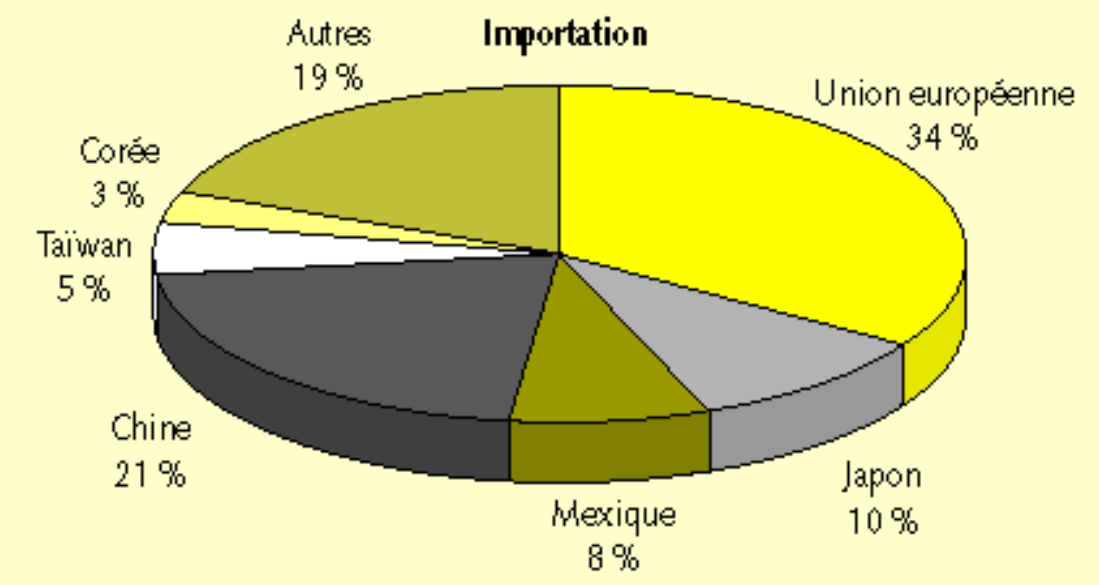

Figure 5. Répartition des flux de soja entre principaux pays en 2000 à l'importation (d'après FAS-USDA). 


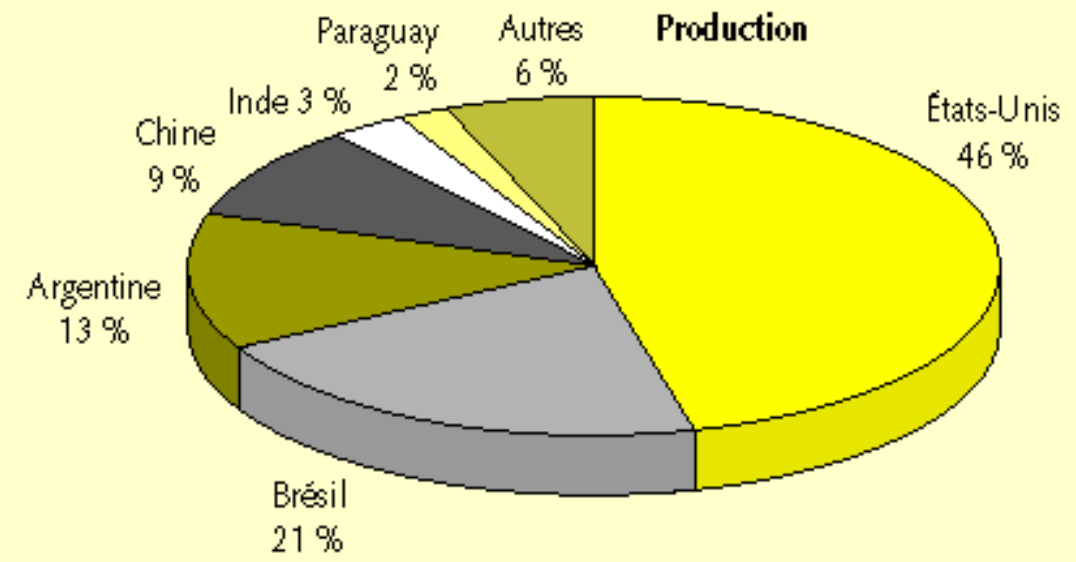

Figure 6. Répartition des flux de soja entre principaux pays en 2000 à la production (d'après FAS-USDA).

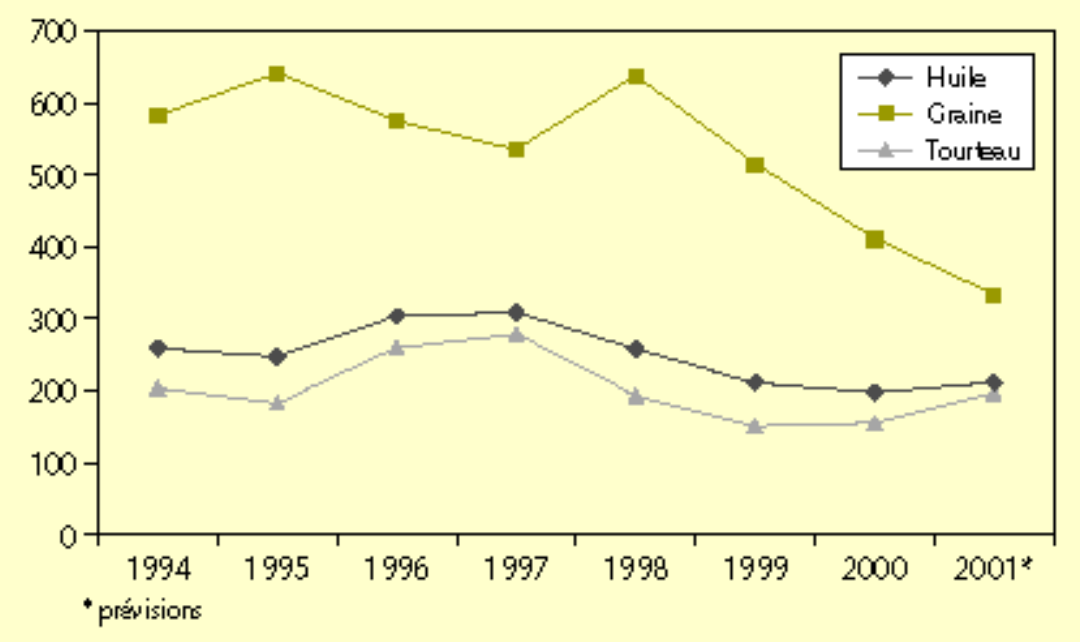

Figure 7. Évolution des prix du soja : prix de la tonne en \$ US à Rotterdam ; CAF pour la graine; FOB pour l'huile et le tourteau (d'après FAS-USDA) 
Tableau 1. Evolution de la production de soja dans les principaux pays (millier de t).

\begin{tabular}{|lrrrrrrrr}
\hline & $\mathbf{1 9 9 0}$ & $\mathbf{1 9 9 4}$ & \multicolumn{1}{c}{$\mathbf{1 9 9 5}$} & \multicolumn{1}{c}{$\mathbf{1 9 9 6}$} & $\mathbf{1 9 9 7}$ & $\mathbf{1 9 9 8}$ & $\mathbf{1 9 9 9}$ & \multicolumn{1}{c}{$\mathbf{2 0 0 \mathbf { 0 } ^ { * }}$} \\
\hline Monde dont: & 108430 & 136420 & 126810 & 130520 & 143400 & 158330 & 158650 & 168540 \\
États-Unis & 52420 & 68500 & 59240 & 64770 & 73180 & 75030 & 72220 & 75380 \\
Brésil & 19890 & 24910 & 25650 & 23560 & 26430 & 31360 & 33200 & 35500 \\
Argentine & 10700 & 11720 & 12130 & 12450 & 11000 & 18720 & 21200 & 24000 \\
Chine & 11010 & 16010 & 13510 & 13230 & 14740 & 15150 & 14290 & 15400 \\
Inde & 2600 & 3930 & 4990 & 5210 & 5350 & 6100 & 5200 & 5500 \\
Paraguay & 1800 & 1800 & 2210 & 2400 & 2670 & 2860 & 2900 & 3100 \\
\hline
\end{tabular}

*Prévision.

Source : FAO/FAS-USDA.

Tableau 2. Evolution des exportations de soja des principaux fournisseurs (millier de t).

\begin{tabular}{|lccccc}
\hline & $\begin{array}{c}\text { 1989/1991 } \\
\text { moyenne }\end{array}$ & $\mathbf{1 9 9 7 / 1 9 9 8}$ & $\mathbf{1 9 9 8 / 1 9 9 9}$ & $\mathbf{1 9 9 9 / 2 0 0 0}$ & $\mathbf{2 0 0 0 / 2 0 0 1 *}$ \\
\hline Monde dont: & 25560 & 38640 & 38670 & 46290 & 47770 \\
Etats-Unis & 16090 & 23690 & 21900 & 26490 & 26130 \\
Brésil & 3570 & 8750 & 8930 & 11160 & 12000 \\
Argentine & 2700 & 3230 & 3230 & 4130 & 5000 \\
Paraguay & 1280 & 2390 & 2300 & 2200 & 2400 \\
\hline
\end{tabular}

* Prévision.

Source : FAO/FAS-USDA.

Tableau 3. Evolution des importations de soja des principaux acheteurs (millier de $t$ ).

\begin{tabular}{|lccccc}
\hline & $\begin{array}{c}\text { 1989/1991 } \\
\text { moyenne }\end{array}$ & $\mathbf{1 9 9 7 / 1 9 9 8}$ & $\mathbf{1 9 9 8 / 1 9 9 9}$ & $\mathbf{1 9 9 9 / 2 0 0 0}$ & 2000/2001* \\
\hline Monde dont: & 25510 & 39940 & 40430 & 47430 & 47510 \\
Union européenne & 12800 & 16880 & 16770 & 15750 & 16650 \\
Chine & - & 2940 & 3850 & 10100 & 10200 \\
Japon & 4450 & 4870 & 4810 & 4900 & 4750 \\
Mexique & 1170 & 3480 & 3760 & 3950 & 4250 \\
Taiwan & - & 2390 & 2150 & 2300 & 2350 \\
Corée & 1040 & 1340 & 1400 & 1550 & 1700 \\
\hline
\end{tabular}

* Prévision.

Soure : FAO/FAS-USDA. 
Tableau 4. Evolution de la trituration dars les principaux pays (millier de t).

\begin{tabular}{|lccccc}
\hline & $\mathbf{1 9 9 2 / 1 9 9 3}$ & $\mathbf{1 9 9 7 / 1 9 9 8}$ & $\mathbf{1 9 9 8 / 1 9 9 9}$ & $\mathbf{1 9 9 9 / 2 0 0 0}$ & $\mathbf{2 0 0 0 / 2 0 0 1 *}$ \\
\hline Monde dont: & 96750 & 126960 & 135810 & 136820 & 143580 \\
Etats-Unis & 34810 & 43460 & 43260 & 42970 & 43270 \\
Brésil & 15550 & 19900 & 21010 & 21200 & 22000 \\
Argentine & 8490 & 13000 & 17510 & 17300 & 18250 \\
Union européenne & 14090 & 16340 & 16230 & 15160 & 16060 \\
Chine & 4490 & 10730 & 12610 & 14870 & 17290 \\
Inde & - & 4770 & 5400 & 4400 & 4750 \\
Mexique & - & 3600 & 3950 & 4100 & 4300 \\
Japon & 3790 & 3720 & 3700 & 3730 & 3720 \\
Taïwan & 2320 & 2070 & 1900 & 1990 & 2050
\end{tabular}

*Prévision.

Source : FAO/FAS-USDA.

Tableau 5. Evolution des exportations de tourteau de soja des principaux fournisseurs (miller de t).

\begin{tabular}{|lccccc}
\hline & $\begin{array}{c}\text { 1989/1991 } \\
\text { moyenne }\end{array}$ & $\mathbf{1 9 9 7 / 1 9 9 8}$ & $\mathbf{1 9 9 8 / 1 9 9 9}$ & $\mathbf{1 9 9 9 / 2 0 0 0}$ & 2000/2001* \\
\hline Monde dont: & 26690 & 36930 & 39060 & 38820 & 39890 \\
Argentine & 5280 & 10230 & 13400 & 13750 & 14400 \\
Brésil & 8720 & 9850 & 10150 & 9520 & 10100 \\
Etats-Unis & 4730 & 8460 & 6460 & 6650 & 6350 \\
Union européenne & 3760 & 4460 & 5040 & 5130 & 5130 \\
Inde & 1100 & 2600 & 2800 & 2350 & 2500 \\
\hline
\end{tabular}

* prévision.

Soure : FAO/FAS-USDA.

Tableau 6. Evolution des importations de tourteau de soja des principaux acheteurs (millier de t).

\begin{tabular}{|lrrrrr}
\hline & $\mathbf{1 9 9 4 / 1 9 9 5}$ & $\mathbf{1 9 9 7 / 1 9 9 8}$ & $\mathbf{1 9 9 8 / 1 9 9 9}$ & $\mathbf{1 9 9 9 / 2 0 0 0}$ & $\mathbf{2 0 0 0 / 2 0 0 1 *}$ \\
\hline Monde dont : & 31430 & 36940 & 39270 & 39260 & 39820 \\
Union européenne & 16800 & 16460 & 19950 & 19770 & 20120 \\
Afrique Nord et Moyen -Orient & 3010 & 3460 & 3640 & 3920 & 4010 \\
Amérique latine & 2550 & 3500 & 3580 & 3700 & 3840 \\
Chine & 50 & 4200 & 1400 & 630 & 500 \\
\hline
\end{tabular}

*Prévision.

Soure : FAS-USDA. 
Tableau 7. Evolution de la consommation de tourteau de soja chez les principaux utilisateurs (millier de t).

\begin{tabular}{|lrrrrr}
\hline & $\mathbf{1 9 9 4 / 1 9 9 5}$ & $\mathbf{1 9 9 7 / 1 9 9 8}$ & $\mathbf{1 9 9 8 / 1 9 9 9}$ & $\mathbf{1 9 9 9 / 2 0 0 0}$ & 2000/2001* \\
\hline Monde dont : & 87470 & 101090 & 107040 & 109640 & 114480 \\
Asie et Océanie & - & 24620 & 26030 & 27930 & 30280 \\
- dont Chine & 5730 & 12760 & 11410 & 12430 & 14220 \\
Etats-Unis & 24080 & 26210 & 27810 & 27560 & 28300 \\
Union européenne & - & 24740 & 27660 & 26820 & 27870 \\
Amérique latine & - & 13340 & 14460 & 15380 & 15930 \\
- dont Brésil & 580 & 5900 & 6650 & 7200 & 7400 \\
Afrique Nord et Moyen -Orient & - & 4460 & 4850 & 5270 & 5530 \\
\hline
\end{tabular}

* Prévision.

Soure : FAS-USDA.

Tableau 8. Evolution des exportations d'huile de soja des principaux fournisseurs (miller de t).

\begin{tabular}{|lccccc}
\hline & $\begin{array}{c}\text { 1989/1991 } \\
\text { moyenne }\end{array}$ & $\mathbf{1 9 9 7 / 1 9 9 8}$ & $\mathbf{1 9 9 8 / 1 9 9 9}$ & $\mathbf{1 9 9 9 / 2 0 0 0}$ & $\mathbf{2 0 0 0 / 2 0 0 1 *}$ \\
\hline Monde dont: & 3700 & 6980 & 8210 & 7220 & 7390 \\
Argentine & 1020 & 2170 & 3140 & 3040 & 3180 \\
Union européenne & 1180 & 1590 & 1700 & 1660 & 1660 \\
Brésil & 730 & 1180 & 1380 & 1130 & 1200 \\
Etats-Unis & 570 & 1400 & 1080 & 620 & 590 \\
\hline
\end{tabular}

*Prévision.

Soure : FAO/FAS-USDA.

Tableau 9. Evolution des importations d'huile de soja des principaux acheteurs (millier de t).

\begin{tabular}{|lccccc}
\hline & $\mathbf{1 9 9 4 / 1 9 9 5}$ & $\mathbf{1 9 9 7 / 1 9 9 8}$ & $\mathbf{1 9 9 8 / 1 9 9 9}$ & $\mathbf{1 9 9 9 / 2 0 0 0}$ & $\mathbf{2 0 0 0 / 2 0 0 1 *}$ \\
\hline Monde dont: & 6130 & 6780 & 7940 & 7020 & 7200 \\
Asie & - & 2880 & 3460 & 2710 & 2730 \\
Amérique latine & - & 1360 & 1840 & 1770 & 1920 \\
Afrique Nord et Moyen-Orient & - & 1230 & 1330 & 1340 & 1390 \\
\hline
\end{tabular}

* Prévision.

Soure : FAO/FAS-USDA. 
Tableau 10. Evolution de la consommation d'huile de soja des principaux utilsateurs (millier de t).

\begin{tabular}{|lccccc}
\hline & $\mathbf{1 9 9 4 / 1 9 9 5}$ & $\mathbf{1 9 9 7 / 1 9 9 8}$ & $\mathbf{1 9 9 8 / 1 9 9 9}$ & $\mathbf{1 9 9 9 / 2 0 0 0}$ & $\mathbf{2 0 0 0 / 2 0 0 1 *}$ \\
\hline Monde dont: & 19460 & 22850 & 24580 & 24390 & 25800 \\
États-Unis & 5860 & 6920 & 7100 & 7280 & 7440 \\
Asie & - & 6270 & 7750 & 7230 & 8010 \\
- dont Chine & 2530 & 3220 & 3080 & 2840 & 3360 \\
- dont Inde & - & 1100 & 1810 & 1580 & 1660 \\
Amérique latine & - & 4650 & 4930 & 5140 & 5380 \\
- dont Brésil & - & 2750 & 2850 & 3000 & 3130 \\
Afrique Nord et Moyen-Orient & - & 1460 & 1830 & 1950 & 2120 \\
Union européenne & - & 1800 & 1830 & 1640 & 1760 \\
\hline
\end{tabular}

* Prévision.

Soure : FAO/FAS-USDA. 